\title{
RAMSEY-TYPE NUMBERS INVOLVING GRAPHS AND HYPERGRAPHS WITH LARGE GIRTH
}

\author{
HIỆP HÀN, TROY RETTER, VOJTĚCH RÖDL, AND MATHIAS SCHACHT
}

\begin{abstract}
A question of Erdős asks if for every pair of positive integers $r$ and $k$, there exists a graph $H$ having girth $(H)=k$ and the property that every $r$-colouring of the edges of $H$ yields a monochromatic cycle $C_{k}$. The existence of such graphs was confirmed by the third author and Ruciński.

We consider the related numerical problem of determining the smallest such graph with this property. We show that for integers $r$ and $k$, there exists a graph $H$ on $R^{10 k^{2}} k^{15 k^{3}}$ vertices (where $R=R\left(C_{k} ; r\right)$ is the $r$-colour Ramsey number for the cycle $C_{k}$ ) having $\operatorname{girth}(H)=k$ and the Ramsey property that every $r$-colouring of $E(H)$ yields a monochromatic $C_{k}$. Two related numerical problems regarding arithmetic progressions in sets and cliques in graphs are also considered.
\end{abstract}

\section{§1. INTRODUCTION}

For an integer $r \geqslant 2$ and graphs $H$ and $F$, we write $H \rightarrow(F)_{r}$ if every $r$-colouring of the edges of $H$ yields a monochromatic copy of $F$. If $H \rightarrow(F)_{r}$, we say that $H$ is Ramsey for $F$ for $r$ colours. It follows from Ramsey's theorem that for every graph $F$ and for every positive integer $r$, there exists a graph $H$ such that $H \rightarrow(F)_{r}$. We consider three Ramsey-type problems that pertain to cycles in graphs and hypergraphs.

1.1. Cycles in Graphs. Our first result relates to a problem suggested by Erdős (see, e.g., [8]), which asks if for every pair of positive integers $r$ and $k$, there exists a graph $H$ having girth $(H)$ at least $k$ and the Ramsey property $H \rightarrow\left(C_{k}\right)_{r}$. The existence of such graphs was first established in [23]. We address the associated numerical problem.

Theorem 1.1. Let $R=R\left(C_{k} ; r\right)$ be the Ramsey number that denotes the least integer $m$ such that $K_{m} \rightarrow\left(C_{k}\right)_{r}$. Then for all integers $k \geqslant 4$ and $r \geqslant 2$, there exists a graph $H$ on $|V(H)|=k^{15 k^{3}} R^{10 k^{2}}$ vertices satisfying girth $(H)=k$ and $H \rightarrow\left(C_{k}\right)_{r}$.

The exponential dependency of $|V(H)|$ on $k$ in Theorem 1.1 is unavoidable. This follows from the observation that a minimal graph $H$ with the desired properties must have

2010 Mathematics Subject Classification. 05C55 (primary), 05D10 (secondary).

Key words and phrases. Ramsey numbers, high girth.

H. Hàn was partly supported by FAPESP (2010/16526-3 and 2013/11353-1). V. Rödl was supported by NSF grant DMS 1301698. M. Schacht was supported through the Heisenberg-Programme of the DFG. 
minimum degree greater than $r$ and girth at least $k$. Note that the $r$-colour Ramsey number $R\left(C_{k} ; r\right)$ is polynomial in $r$ for fixed even $k$, while for fixed odd $k$ it satisfies the exponential relation $c_{1}^{r} \leqslant R\left(C_{k} ; r\right) \leqslant c_{2}^{r \log r}$ for some positive constants $c_{1}$ and $c_{2}{ }^{1}$ (see, e.g., [10]). This leads to the following corollary, which shows that the additional girth requirement on $H$ leads to a Ramsey graph of order comparable to $R\left(C_{k}, r\right)$.

Corollary 1.2. For every integer $k \geqslant 3$, there exist constants $K_{e}$ and $K_{o}$ such that for every integer $r \geqslant 2$, there exists a graph $H$ such that $\operatorname{girth}(H)=k$ and $H \rightarrow\left(C_{k}\right)_{r}$, which satisfies $|V(H)| \leqslant r^{K_{e}}$ if $k$ is even and $|V(H)| \leqslant K_{o}^{r \log r}$ if $k$ is odd.

In Section 6, we will further expand upon Theorem 1.1. In particular, we prove a lower bound and give a simpler proof for the cases $k=4$ and $k=6$.

1.2. Arithmetic Progressions. For a subset $S \subseteq \mathbb{N}$ and integers $k \geqslant 3$ and $r \geqslant 2$, we write $S \rightarrow\left(A P_{k}\right)_{r}$ to signify that every $r$-colouring of the integers in $S$ yields a monochromatic arithmetic progression of length $k$. Van der Waerden's theorem shows for all integers $k \geqslant 3$ and $r \geqslant 2$ that there is some integer $N$ such that $[N] \rightarrow\left(A P_{k}\right)_{r}$, where we denote by $[N]$ the set of the first $N$ positive integers $\{1,2, \ldots, N\}$. Several refinements of this well known theorem have been considered. One generalisation, suggested by Erdős [9], asks if for all $k \geqslant 3$ and $r \geqslant 2$, there exists an $A P_{k+1}$-free set $S \subseteq \mathbb{N}$ that has the property $S \rightarrow\left(A P_{k}\right)_{r}$, where a set is $A P_{k+1}$-free if it does not contain an arithmetic progression of length $k+1$. This was answered independently by Spencer [28] and by Nešetřil and Rödl [19]. Moreover, Graham and Nešetřil [15] showed that there exist arbitrarily large $A P_{k+1}$-free sets $S$ that have the property $S \rightarrow\left(A P_{k}\right)_{r}$ and are minimal in the sense that, for every $s \in S$, the subset $S^{\prime}=S \backslash\{s\}$ fails have the van der Waerden property, i.e., $S^{\prime} \rightarrow\left(A P_{k}\right)_{r}$.

Furthermore, one may want to restrict the structure of the arithmetic progressions of length $k$ in a set $S \subseteq \mathbb{N}$, but keep the van der Waerden property. That is, consider the system of copies of arithmetic progression of length $k$ in $S$, which is the $k$-uniform hypergraph $\left(S,\left(\begin{array}{c}S \\ A P_{k}\end{array}\right)\right)$ on the vertex set $S$ with edge set $\left(\underset{A P_{k}}{S}\right)$ consisting of the $k$ element subsets of $S$ that form arithmetic progressions of length $k$. For a simpler notation, it will be convenient to identify this hypergraph just by its edge set. Moreover, we denote its chromatic number simply by $\chi\left(\begin{array}{c}S \\ A P_{k}\end{array}\right)$ instead of $\chi\left(\left(\begin{array}{c}S \\ A P_{k}\end{array}\right)\right)$. Similarly, we suppress the outer pair of parentheses for other numerical hypergraph parameters as well.

Observe that $S \rightarrow\left(A P_{k}\right)_{r}$ if and only if the chromatic number satisfies $\chi\left(\underset{A P_{k}}{S}\right)>r$. Hence, van der Waerden's theorem establishes that for fixed $k$, the chromatic number $\chi\left(\begin{array}{c}{[N]} \\ A P_{k}\end{array}\right) \rightarrow \infty$

\footnotetext{
${ }^{1}$ We denote by log the binary logarithm and by $\ln$ the natural logarithm.
} 
as $N$ tends to infinity. In view of the result of Erdős and Hajnal [11], which establishes the existence of hypergraphs having both large chromatic number and large girth, it is naturally to ask the following. Does for all $k, g \geqslant 3$, and $r \geqslant 2$ there exist a set $S \subseteq \mathbb{N}$ so that the hypergraph $\left(\begin{array}{c}S \\ A P_{k}\end{array}\right)$ satisfies both the properties

(P1) $\chi\left(\begin{array}{c}S \\ A P_{k}\end{array}\right)>r$

(P2) $\operatorname{girth}\left(\begin{array}{c}S \\ A P_{k}\end{array}\right) \geqslant g ?$

As usual we say a $k$-uniform hypergraph has girth at least $g$ if, for any integer $h$ with $2 \leqslant h<g$, any subset of $h$ edges span at least $(k-1) h+1$ vertices. In particular, $\operatorname{girth}\left(\begin{array}{c}S \\ A P_{k}\end{array}\right) \geqslant 3$ implies that no two arithmetic progressions can intersect in more than one point, which implies that $S$ is $A P_{k+1}$-free. The existence of sets $S \subseteq \mathbb{N}$ satisfying properties (P1) and (P2) was established in [22] (see also [23]) and our next result gives an upper bound for the size of the smallest such set $S$.

Theorem 1.3. Let $W=\operatorname{vdW}(k, r)$ denote the least integer $N$ such that $[N] \rightarrow\left(A P_{k}\right)_{r}$. Then for all integers $k, g \geqslant 3$, and $r \geqslant 2$, there exists a set $S \subseteq \mathbb{N}$ such that

$$
\chi\left(\begin{array}{c}
S \\
A P_{k}
\end{array}\right)>r, \quad \operatorname{girth}\left(\begin{array}{c}
S \\
A P_{k}
\end{array}\right) \geqslant g, \quad \text { and } \quad|S| \leqslant k^{40 k^{2}(k+g)} W^{12 k(k+g)} .
$$

To illustrate the result, consider the special case $k=3$ for fixed $g \geqslant 3$. A result of Sanders [25] (see also [3]) implies that $\operatorname{vdW}(3 ; r) \leqslant \exp \left(r^{1+o(1)}\right)$, where the error term $o(1) \rightarrow 0$ as $r \rightarrow \infty$. Consequently, our result yields the existence of a set $S$ of size at most $\exp \left(r^{1+o(1)}\right)$ such that the properties $S \rightarrow\left(A P_{3}\right)_{r}$ and $\left(\begin{array}{c}S \\ A P_{3}\end{array}\right) \geqslant g$ both hold. In other words, as in Theorem 1.1 the added girth condition does not essentially increase the best known upper bound in this case.

1.3. Cliques in Graphs. Another well known problem of Erdős and Hajnal [12] asked if, for every pair of positive integers $k$ and $r$, there exists a $K_{k+1}$-free graph $H$ such that $H \rightarrow\left(K_{k}\right)_{r}$. The case $r=2$ was confirmed by Folkman [14], and the general case $r>2$ was resolved by Nešetřil and Rödl [18]. Subsequently, Erdős [8] asked for a strengthened form of this result, namely the existence of a graph $H$ with $H \rightarrow\left(K_{k}\right)_{r}$ in which no two copies of $K_{k}$ share more than one edge, which was established in [20] (see also [21] for a generalisation from cliques $K_{k}$ to arbitrary graphs).

As in the context of van der Waerden's theorem in Section 1.2, we may consider the structure of the cliques in $H$ in more detail, i.e., we consider the system of copies of $K_{k}$ in $H$, which is the $\left(\begin{array}{l}k \\ 2\end{array}\right)$-uniform hypergraph $\left(E(H),\left(\begin{array}{c}H \\ K_{k}\end{array}\right)\right)$ having vertex set $E(H)$ and hyperedges corresponding to the edge sets of copies of $K_{k}$ in $H$. As above we identify this hypergraph by its edge set $\left(\begin{array}{c}H \\ K_{k}\end{array}\right)$ and denote by $\chi\left(\begin{array}{c}H \\ K_{k}\end{array}\right)$ and $\operatorname{girth}\left(\begin{array}{c}H \\ K_{k}\end{array}\right)$ its chromatic number and its girth. Again the statement $H \rightarrow\left(K_{k}\right)_{r}$ is equivalent to $\chi\left(\begin{array}{c}H \\ K_{k}\end{array}\right)>r$ and the property 
that any two copies of $K_{k}$ in $H$ share at most one edge is equivalent to $\operatorname{girth}\left(\begin{array}{c}H \\ K_{k}\end{array}\right) \geqslant 3$. We give a new proof of the result from [20] that leads to a new upper bound on the size of the smallest such $H$.

Theorem 1.4. Let $R=R\left(K_{k} ; r\right)$ be the Ramsey number that denotes the least integer $m$ such that $K_{m} \rightarrow\left(K_{k}\right)_{r}$. Then for all integers $k, g \geqslant 3$, and $r \geqslant 2$, there exists a graph $H$ such that

$$
\chi\left(\begin{array}{c}
H \\
K_{k}
\end{array}\right)>r, \quad \operatorname{girth}\left(\begin{array}{c}
H \\
K_{k}
\end{array}\right) \geqslant g, \quad \text { and } \quad|V(H)| \leqslant k^{40 g k^{4}} R^{40 g k^{2}} .
$$

By reversing the dependency between $g$ and $|V(H)|$, we obtain the following corollary.

Corollary 1.5. For all integers $k \geqslant 3$ and $r \geqslant 2$, there exist $c_{k, r}>0$ and $n_{0}$ such that, for every integer $n \geqslant n_{0}$, there exists a graph $H$ on $n$ vertices satisfying both $H \rightarrow\left(K_{k}\right)_{r}$ and girth $\left(\begin{array}{c}H \\ K_{k}\end{array}\right) \geqslant c_{k, r} \log n$.

It can be shown that any graph $H$ on $n$ vertices satisfying $H \rightarrow\left(K_{k}\right)_{r}$ must also

satisfy girth $\left(\begin{array}{c}H \\ K_{k}\end{array}\right)=O(\log n)$, due to the degree condition required by $\chi\left(\begin{array}{c}H \\ K_{k}\end{array}\right)>r$ and, in that sense, our result gives an optimal order of magnitude for the girth.

1.4. Organization. In Section 2 we state the so-called Container Lemma, which playes a central rôle in our proofs. The details of the proofs of Theorems 1.1, 1.3, and 1.4 are given in Sections 3, 4, and 5 respectively. Section 6 contains some remarks related to Theorem 1.1.

\section{§2. Hypergraph Containers}

The proofs of the theorems presented in Section 1 use some ideas from $[17,24]$ and rely on random constructions combined with the Container Method of Balogh, Morris, and Samotij [1] and of Saxton and Thomason [26]. For the numerical aspects the container result from [26] seemed to be better suited and we state it below (see Theorem 2.1).

Roughly speaking, this lemma states that, if a given hypergraph $\mathcal{H}$ satisfies some numerical 'degree conditions', then there there exists a relatively 'small' family of sets of so-called 'containers' (sets $\mathcal{C}$ in Theorem 2.1 below) that are 'almost' independent sets of vertices that cover all independent sets of $\mathcal{H}$.

We now introduce the notation necessary for the formulation of this theorem. For a $h$-uniform hypergraph $\mathcal{H}$, let $e(\mathcal{H})$ denote the number of (hyper)edges in $\mathcal{H}$. For a set $J \subseteq V(\mathcal{H})$ we define the degree of $J$ by

$$
d(J)=|\{e \in E(\mathcal{H}): e \supseteq J\}|
$$

and for $j=1, \ldots, h$ we define the maximum $j$-degree of a vertex $v \in V(\mathcal{H})$ by 


$$
d_{j}(v)=\max \left\{d(J): J \in\left(\begin{array}{c}
V(H) \\
j
\end{array}\right) \text { and } v \in J\right\} .
$$

The average of $d_{j}(v)$ is denoted by

$$
d_{j}=\frac{1}{|V(\mathcal{H})|} \sum_{v \in V} d_{j}(v) .
$$

Note that $d_{1}(v)$ is just the degree of $v$ in $\mathcal{H}$ and, consequently, $d_{1}$ denotes the average vertex degree of $\mathcal{H}$. With this notation at hand we state the Container Lemma from [26, Corollary 3.6].

Theorem 2.1 (Container Lemma). Let $\mathcal{H}=(V, E)$ be a h-uniform hypergraph and suppose $\tau, \varepsilon \in(0,1 / 2)$ satisfy

$$
\frac{6 \cdot h ! \cdot 2^{\left(\begin{array}{c}
h \\
2
\end{array}\right)}}{d_{1}} \sum_{j=2}^{h} \frac{d_{j}}{2^{\left(\begin{array}{c}
j-1 \\
2
\end{array}\right)} \tau^{j-1}} \leqslant \varepsilon .
$$

Then for integers

$$
K=800 h(h !)^{3} \quad \text { and } \quad s=\lfloor K \log (1 / \varepsilon)\rfloor
$$

the following holds.

For every independent set $I \subseteq V$ in $\mathcal{H}$, there exists an $s$-tuple $\mathcal{S}=\left(\mathcal{S}_{1}, \ldots, \mathcal{S}_{s}\right)$ of subsets of $V$ and a subset $\mathcal{C}=\mathcal{C}(\mathcal{S}) \subseteq V$ only depending on $\mathcal{S}$ such that

(i) $\bigcup_{i \in[s]} \mathcal{S}_{i} \subseteq I \subseteq \mathcal{C}$,

(ii) $e(\mathcal{C}) \leqslant \varepsilon \cdot e(\mathcal{H})$, and

(iii) for every $i \in[s]$ we have $\left|\mathcal{S}_{i}\right| \leqslant \tau K|V|$.

The Container Lemma stated here is an abridged version of [26, Corollary 3.6], which suffices for our purpose. For the explicit constant 800 appearing in the constant $K$ see the discussion following Corollary 3.6 in [26].

\section{§3. Proof of TheOrem 1.1}

In this section we prove Theorem 1.1. For that we have to show that there exists a graph $H$ on at most $k^{15 k^{3}} R^{10 k^{2}}$ vertices (where $R=R\left(C_{k} ; r\right)$ is the $r$-colour Ramsey number for $C_{k}$ ) with $\operatorname{girth}(H)=k$ and the Ramsey property $H \rightarrow\left(C_{k}\right)_{r}$.

Proof. For integers $k \geqslant 4$ and $r \geqslant 2$ let $R=R\left(C_{k} ; r\right)$ be the $r$-colour Ramsey number for $C_{k}$. We first define all constants involved in the proof. For the application of the Container Lemma we set

$$
\varepsilon=\frac{1}{r R^{k}} \quad \text { and } \quad D_{\tau}=\frac{2^{2 k}}{\varepsilon^{1 /(k-1)}}
$$


and we fix integers

$$
K=800 k(k !)^{3}<30 k^{3 k} \quad \text { and } \quad s=\lfloor K \log (1 / \varepsilon)\rfloor<30 k^{3 k} \log \left(r R^{k}\right) .
$$

We set

$$
D_{p}=10 R^{2} r^{2} s^{2} K D_{\tau} \log \left(10 R^{2} r\right)<k^{15 k} R^{10}
$$

and define

$$
n=D_{p}^{k^{2}}<k^{15 k^{3}} R^{10 k^{2}} .
$$

Finally, we define the following parameters appearing in the proof

$$
\tau=D_{\tau} n^{-\frac{k-2}{k-1}} \quad \text { and } \quad p=D_{p} n^{-\frac{k-2}{k-1}} .
$$

In the proof we consider the binomial random graph $G(n, p)$ and the theorem is a consequence of the following two claims, which we verify below.

Claim 3.1. $\mathbb{P}(\operatorname{girth}(G(n, p)) \geqslant k) \geqslant \exp \left(-k D_{p}^{k-1} n\right)$.

Claim 3.2. $\mathbb{P}\left(G(n, p) \rightarrow\left(C_{k}\right)_{r}\right) \geqslant 1-\exp \left(-\frac{p}{2 R^{2}}\left(\begin{array}{l}n \\ 2\end{array}\right)\right)$.

In fact, since our choice of constants guarantees $p(n-1)>4 R^{2} k D_{p}^{k-1}$, it follows from Claims 3.1 and 3.2 that with positive probability the random graph $G(n, p)$ has girth at least $k$ and the Ramsey-property for $C_{k}$ and $r$ colours. Consequently, Theorem 1.1 follows from our choice of $n$ in (5).

Proof of Claim 3.1. For the lower bound of the probability that $G(n, p)$ has girth at least $k$, we will use the FKG-inequality (see, e.g., [16, Section 2.2]). For this purpose, let $X_{k-1}$ be the random variable of the number of cycles of length less than $k$. Clearly, $X_{k-1}$ is the sum of monotone increasing indicator random variables and, hence, the FKG-inequality asserts

$$
\mathbb{P}(\operatorname{girth}(G(n, p)) \geqslant k)=\mathbb{P}\left(X_{k-1}=0\right) \stackrel{\mathrm{FKG}}{\geqslant} \prod_{j=3}^{k-1}\left(1-p^{j}\right)^{\frac{(j-1) !}{2}\left(\begin{array}{l}
n \\
j
\end{array}\right)} \geqslant \exp \left(-\frac{\mathbb{E}\left[X_{k-1}\right]}{1-p^{3}}\right),
$$

where we used the estimate $1-x \geqslant \exp (-x /(1-x))$ for the last inequality. Since $p n \geqslant 1$ we have

$$
\mathbb{E}\left[X_{k-1}\right]=\sum_{j=3}^{k-1} \frac{(j-1) !}{2}\left(\begin{array}{c}
n \\
j
\end{array}\right) p^{j} \leqslant \sum_{j=3}^{k-1} \frac{(p n)^{j}}{2 j} \leqslant \frac{k}{6}(p n)^{k-1} \stackrel{(6)}{=} \frac{k}{6} D_{p}^{k-1} n
$$

and the claim follows from $1-p^{3}>1 / 6$ and (7).

Proof of Claim 3.2. We will apply the Container Lemma to the $k$-uniform hypergraph $\mathcal{H}=\left(\begin{array}{l}K_{n} \\ C_{k}\end{array}\right)$ which is the system of all cycles $C_{k}$ of length $k$ in $K_{n}$, i.e., $V(\mathcal{H})=E\left(K_{n}\right)$ and $k$ 
edges of $K_{n}$ correspond to a hyperedge in $\mathcal{H}$, if they form a cycle of length $k$. For the application of Theorem 2.1 we first verify (1). In that direction we note

$$
d_{1}=\frac{k \cdot|E(\mathcal{H})|}{|V(\mathcal{H})|}=\frac{k !\left(\begin{array}{l}
n \\
k
\end{array}\right)}{2\left(\begin{array}{l}
n \\
2
\end{array}\right)} \geqslant \frac{k !}{k^{k}} n^{k-2}, \quad d_{j} \leqslant n^{k-j-1}
$$

for $j=2, \ldots, k-1$, and $d_{k}=1$. Therefore, for $j=2, \ldots, k-1$ we have

$$
\frac{d_{j}}{d_{1} \cdot \tau^{j-1}} \leqslant \frac{k^{k}}{k ! \cdot(\tau n)^{j-1}} \leqslant \frac{k^{k}}{k ! \cdot \tau n}=\frac{k^{k}}{k ! \cdot D_{\tau} n^{1 /(k-1)}} \leqslant \frac{k^{k}}{k ! \cdot n^{1 /(k-1)}}
$$

and, moreover,

$$
\frac{d_{k}}{d_{1} \cdot \tau^{k-1}} \leqslant \frac{k^{k}}{k ! n^{k-2} \cdot \tau^{k-1}}=\frac{k^{k}}{k ! \cdot D_{\tau}^{k-1}} .
$$

Combining both estimates yields

$$
\frac{6 \cdot k ! \cdot 2^{\left(\begin{array}{c}
k \\
2
\end{array}\right)}}{d_{1}} \sum_{j=2}^{k} \frac{d_{j}}{2^{\left(\begin{array}{c}
j-1 \\
2
\end{array}\right)} \tau^{j-1}} \leqslant \frac{6 \cdot k^{k+1} \cdot 2^{\left(\begin{array}{c}
k \\
2
\end{array}\right)}}{\min \left\{n^{1 /(k-1)}, D_{\tau}^{k-1}\right\}} \stackrel{(2),(5)}{\leqslant} \varepsilon .
$$

Having verified (1) of Theorem 2.1, we infer properties $(i)-($ iii $)$ for every independent set $I \subseteq V(\mathcal{H})$. We consider the family $\mathcal{B}$ of all graphs $B \subseteq K_{n}$ that fail to have the Ramsey property, i.e., $B \nrightarrow\left(C_{k}\right)_{r}$. Below we establish Claim 3.2 by showing

$$
\mathbb{P}(G(n, p) \in \mathcal{B}) \leqslant \exp \left(-\frac{p}{2 R^{2}}\left(\begin{array}{l}
n \\
2
\end{array}\right)\right) .
$$

By the definition of $\mathcal{B}$, for every $B \in \mathcal{B}$ there exists a partition $E(B)=I_{1}^{B} \cup \ldots \cup I_{r}^{B}$ with the property that none of the sets $I_{i}^{B}$ contains a cycle $C_{k}$. In particular, each $I_{i}^{B}$ is an independent set in $\mathcal{H}$ and, therefore, properties $(i)-(i i i)$ of the Container Lemma assert that for every $i \in[r]$ there exists an $s$-tuple $\mathcal{S}_{i}^{B}=\left(\mathcal{S}_{i, 1}^{B}, \ldots, \mathcal{S}_{i, s}^{B}\right)$ of subsets of $I_{i}^{B}$ and a container set $\mathcal{C}\left(\mathcal{S}_{i}^{B}\right) \supseteq I_{i}^{B}$ such that

$$
\left|\mathcal{S}_{i, \sigma}^{B}\right| \leqslant \tau K\left(\begin{array}{l}
n \\
2
\end{array}\right)
$$

for every $\sigma \in[s]$ and

$$
\left|e\left(\mathcal{C}\left(\mathcal{S}_{i}^{B}\right)\right)\right| \leqslant \varepsilon \cdot e(\mathcal{H})=\varepsilon \cdot \frac{(k-1) !}{2}\left(\begin{array}{l}
n \\
k
\end{array}\right) .
$$

We also set $\mathscr{S}^{B}=\left(\mathcal{S}_{1}^{B}, \ldots, \mathcal{S}_{r}^{B}\right)$ and $\mathscr{C}^{B}=\left(\mathcal{C}\left(\mathcal{S}_{1}^{B}\right), \ldots, \mathcal{C}\left(\mathcal{S}_{r}^{B}\right)\right)$.

Moreover, for any possible $r$-tuple $\mathscr{S}=\left(\mathcal{S}_{1}, \ldots, \mathcal{S}_{r}\right)$ of $s$-tuples of sets of size at most $\tau K\left(\begin{array}{l}n \\ 2\end{array}\right)$ we consider the corresponding container vector $\mathscr{C}(\mathscr{S})=\left(\mathcal{C}\left(\mathcal{S}_{1}\right), \ldots, \mathcal{C}\left(\mathcal{S}_{r}\right)\right)$ given by the Container Lemma. We denote by $D(\mathscr{S})$ its complement in $E\left(K_{n}\right)$ given by

$$
D(\mathscr{S})=E\left(K_{n}\right) \backslash\left(\mathcal{C}\left(\mathcal{S}_{1}\right) \cup \cdots \cup \mathcal{C}\left(\mathcal{S}_{r}\right)\right) .
$$

We observe that for any $B \in \mathcal{B}$ the following two properties hold:

(a) $\bigcup_{i \in[r]} \bigcup_{\sigma \in[s]} \mathcal{S}_{i, \sigma}^{B} \subseteq E(B)$ and 
(b) $E(B) \cap D\left(\mathscr{S}^{B}\right)=\varnothing$, since

$$
E(B)=I_{1}^{B} \cup \ldots \cup I_{r}^{B} \subseteq \mathcal{C}\left(\mathcal{S}_{1}^{B}\right) \cup \cdots \cup \mathcal{C}\left(\mathcal{S}_{r}^{B}\right)=E\left(K_{n}\right) \backslash D\left(\mathscr{S}^{B}\right)
$$

From $(a)$ and $(b)$ we infer that

$$
\begin{aligned}
\mathbb{P}(G(n, p) \in \mathcal{B}) & \leqslant \sum_{\mathscr{S}=\left(\mathcal{S}_{1}, \ldots, \mathcal{S}_{r}\right)} p^{\left|\bigcup_{i \in[r]} \bigcup_{\sigma \in[s]} \mathcal{S}_{i, \sigma}\right|} \cdot \mathbb{P}(E(G(n, p)) \cap D(\mathscr{S})=\varnothing) \\
& \leqslant \max _{\mathscr{S}} \mathbb{P}(E(G(n, p)) \cap D(\mathscr{S})=\varnothing) \sum_{\mathscr{S}} p\left|\cup_{i \in[r]} \bigcup_{\sigma \in[s]} \mathcal{S}_{i, \sigma}\right|
\end{aligned}
$$

where the sum and the maximum are taken over all $r$-tuples $\mathscr{S}=\left(\mathcal{S}_{1}, \ldots, \mathcal{S}_{r}\right)$ of $s$-tuples $\mathcal{S}_{i}=\left(\mathcal{S}_{i, 1}, \ldots, \mathcal{S}_{i, s}\right)$ of sets $\mathcal{S}_{i, \sigma}$ of size at most $\tau K\left(\begin{array}{l}n \\ 2\end{array}\right)$ for $i \in[r]$ and $\sigma \in[s]$. We will use property $(i i)$ of the Container Lemma to bound the maximum probability, while our choice of constants allow us to derive a sufficient bound for the sum.

For the maximum probability below we first observe that for every $\mathscr{S}=\left(\mathcal{S}_{1}, \ldots, \mathcal{S}_{r}\right)$ we have

$$
|D(\mathscr{S})|>\frac{1}{R^{2}}\left(\begin{array}{l}
n \\
2
\end{array}\right)
$$

For the proof we use the fact that for any $(r+1)$-colouring of $E\left(K_{n}\right)$ either there are more than

$$
\frac{1}{2\left(\begin{array}{l}
R \\
k
\end{array}\right)}\left(\begin{array}{l}
n \\
k
\end{array}\right)
$$

monochromatic copies of $C_{k}$ in the first $r$ colours or there are more than $\frac{1}{R^{2}}\left(\begin{array}{l}n \\ 2\end{array}\right)$ edges having the last colour (see, e.g., [24, Proposition 8] for the same assertion for cliques $K_{k}$ instead of cycles).

In view of this fact, we consider $\mathcal{C}\left(\mathcal{S}_{1}\right) \cup \cdots \cup \mathcal{C}\left(\mathcal{S}_{r}\right) \cup D(\mathscr{S})$ as an $(r+1)$-colouring of $E\left(K_{n}\right)$. Owing to property (ii) of the Container Lemma (see (8)), every $\mathcal{C}\left(\mathcal{S}_{i}\right)$ contains at most $\varepsilon \frac{(k-1) !}{2}\left(\begin{array}{l}n \\ k\end{array}\right)$ copies of $C_{k}$ and, hence, there are at most

$$
r \cdot \varepsilon \frac{(k-1) !}{2}\left(\begin{array}{l}
n \\
k
\end{array}\right) \stackrel{(2)}{\leqslant} \frac{1}{2\left(\begin{array}{l}
R \\
k
\end{array}\right)}\left(\begin{array}{l}
n \\
k
\end{array}\right)
$$

monochromatic copies in the first $r$ colours. Therefore, the mentioned fact above yields (10) and, consequently, we arrive at

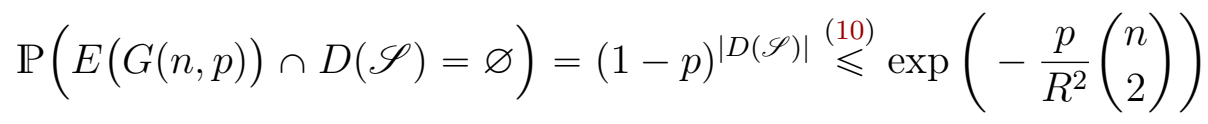

for every $\mathscr{S}$ considered here. In particular, (11) bounds the maximum probability considered in the R-H-S of (9) and below we turn to the sum in (9). 
Owing to $\left|\mathcal{S}_{i, \sigma}\right| \leqslant \tau K\left(\begin{array}{l}n \\ 2\end{array}\right)$ for every $i \in[r]$ and $\sigma \in[s]$ we have

$$
\left.\sum_{\mathscr{S}=\left(\mathcal{S}_{1}, \ldots, \mathcal{S}_{r}\right)} p^{\left|\bigcup_{i \in[r]} \bigcup_{\sigma \in[s]} \mathcal{S}_{i, \sigma}\right|} \leqslant \sum_{m=0}^{r \cdot s \cdot \tau K\left(\begin{array}{l}
n \\
2
\end{array}\right)}\left(\begin{array}{c}
n \\
2
\end{array}\right)\right) 2^{r s m} p^{m} \leqslant \sum_{m=0}^{r \cdot s \cdot \tau K\left(\begin{array}{l}
n \\
2
\end{array}\right)}\left(\frac{\mathrm{e}\left(\begin{array}{l}
n \\
2
\end{array}\right)}{m} 2^{r s} p\right)^{m} .
$$

Since the function $m \mapsto\left(\mathrm{e}\left(\begin{array}{l}n \\ 2\end{array}\right) 2^{r s} p / m\right)^{m}$ is unimodal and attains its maximum value for $m_{0}=\left(\begin{array}{l}n \\ 2\end{array}\right) 2^{r s} p \geqslant \operatorname{rs\tau } K\left(\begin{array}{l}n \\ 2\end{array}\right)$, we can bound the summands in R-H-S above by the last one and obtain

$$
\begin{aligned}
\sum_{\mathscr{S}=\left(\mathcal{S}_{1}, \ldots, \mathcal{S}_{r}\right)} p^{\left|\cup_{i \in[r]} \bigcup_{\sigma \in[s]} \mathcal{S}_{i, \sigma}\right|} & \leqslant\left(r s \tau K\left(\begin{array}{l}
n \\
2
\end{array}\right)+1\right) \cdot\left(\frac{\mathrm{e}\left(\begin{array}{l}
n \\
2
\end{array}\right)}{r s \tau K\left(\begin{array}{c}
n \\
2
\end{array}\right)} 2^{r s} p\right)^{r s \tau K\left(\begin{array}{c}
n \\
2
\end{array}\right)} \\
& \leqslant n^{2} \cdot\left(\frac{2^{r s} D_{p}}{r s K D_{\tau}}\right)^{r s \tau K\left(\begin{array}{l}
n \\
2
\end{array}\right)} \\
& \stackrel{(4)}{=} n^{2} \cdot\left(2^{r s} \mathrm{e} \cdot 10 R^{2} r s \log \left(10 R^{2} r\right)\right)^{r s \tau K\left(\begin{array}{l}
n \\
2
\end{array}\right)} \\
& \leqslant n^{2} \cdot \exp \left(r s \tau K\left(\begin{array}{l}
n \\
2
\end{array}\right)\left(r s+1+\ln \left(10 R^{2} r s \log \left(10 R^{2} r\right)\right)\right)\right) \\
& \stackrel{(4)}{\leqslant} n^{2} \cdot \exp \left(\frac{p}{3 R^{2}}\left(\begin{array}{l}
n \\
2
\end{array}\right)\right) \\
& \leqslant \exp \left(\frac{p}{2 R^{2}}\left(\begin{array}{l}
n \\
2
\end{array}\right)\right) .
\end{aligned}
$$

Finally, combining (11) and (12) with (9) leads to

$$
\mathbb{P}(G(n, p) \in \mathcal{B}) \leqslant \exp \left(-\frac{p}{R^{2}}\left(\begin{array}{l}
n \\
2
\end{array}\right)\right) \cdot \exp \left(\frac{p}{2 R^{2}}\left(\begin{array}{l}
n \\
2
\end{array}\right)\right)=\exp \left(-\frac{p}{2 R^{2}}\left(\begin{array}{l}
n \\
2
\end{array}\right)\right),
$$

which concludes the proof of the claim.

\section{§4. Proof of Theorem 1.3}

We prove Theorem 1.3 by establishing that, for given integers $k \geqslant 3, r \geqslant 2$, and $g \geqslant 2$, there exists a set $S \subseteq \mathbb{N}$ of size at most $k^{40 k^{2}(k+g)} W^{12 k(k+g)}$ (where $W=\operatorname{vdW}(k ; r)$ is the van der Waerden number guaranteeing monochromatic arithmetic progressions of length $k$

for any $r$-colouring of $[W])$ such that the hypergraph $\left(\begin{array}{c}S \\ A P_{k}\end{array}\right)$ has chromatic number greater than $r$, and girth at least $g$.

Proof. Let $k \geqslant 3, r \geqslant 2$, and $g \geqslant 2$ be given and let $W=\operatorname{vdW}(k ; r)$ be the van der Waerden number. We first define all constants involved in the proof. For the application of the Container Lemma we set

$$
\varepsilon=\frac{1}{r W^{3}} \quad \text { and } \quad D_{\tau}=\left(\frac{6 \cdot k ! \cdot 2^{\left(\begin{array}{c}
k \\
2
\end{array}\right) \cdot k^{3}}}{\varepsilon}\right)^{\frac{1}{k-1}}
$$


and we fix integers

$$
K=800 k(k !)^{3}<30 k^{3 k} \quad \text { and } \quad s=\lfloor K \log (1 / \varepsilon)\rfloor<30 k^{3 k} \log \left(r W^{3}\right) .
$$

We set

$$
D_{p}=128 W r^{2} s^{2} K D_{\tau} \log (128 W r)<2^{40} k^{10 k} r^{3} W^{3}
$$

and define

$$
n=k^{4 g} D_{p}^{2 k(k+g)}<k^{40 k^{2}(k+g)} W^{12 k(k+g)} .
$$

Finally, we define the following parameters appearing in the proof

$$
\tau=D_{\tau} n^{-\frac{1}{k-1}}, \quad p=D_{p} n^{-\frac{1}{k-1}}, \quad \text { and } \quad t=\frac{p n}{8 W} .
$$

Let $[n]_{p}$ denote the random set obtained by choosing each element of $[n]=\{1,2, \ldots, n\}$ independently with probability $p$. The theorem is an immediate consequence of the following two claims.

Claim 4.1. With probability larger than $1 / 2$ for the random subset $[n]_{p}$ there exists a set $T \subseteq[n]_{p}$ of size at most $t$ such that such that girth $\left(\begin{array}{c}{[n]_{p} \backslash T} \\ A P_{k}\end{array}\right) \geqslant g$.

Claim 4.2. With probability larger than $1 / 2$ the random subset $[n]_{p}$ satisfies $\chi\left(\begin{array}{c}{[n]_{p} \backslash T} \\ A P_{k}\end{array}\right)>r$ for every subset $T \subseteq[n]_{p}$ of size at most $t$.

Together, these claims establish that, with positive probability, the random set $[n]_{p}$ will have the property that there exists a set $T \subseteq[n]_{p}$ of size $t$ so that the hypergraph $\left(\begin{array}{c}{[n]_{p} \backslash T} \\ A P_{k}\end{array}\right)$ has girth at least $g$ and chromatic number bigger than $r$. Thus, these claims together with our choice of $n$ in (16) establish the existence of a set $S \subseteq \mathbb{N}$ as claimed in Theorem 1.3. We remark that, such a set will likely have only $O(p n)$ elements (not $n$ elements). However, this improvement is negligible.

Proof of Claim 4.1. The proof follows by a standard first moment argument. Recall that a 2-cycle in a hypergraph consists of two hyperedges sharing at least two vertices and for $j>2$ a $j$-cycle consists of a cyclically ordered sequence of hyperedges $e_{1}, e_{2}, \ldots, e_{j}$ where the intersection of two consecutive edges is exactly 1 , the intersection of any two nonconsecutive edges is empty, and the intersection points for each pair of consecutive edges is unique (which for $j \geqslant 4$ is already implied by the other two conditions). Let the random variable $X_{j}$ denote the number of $j$-cycles appearing in the random hypergraph $\left(\begin{array}{c}{[n]_{p}} \\ A P_{k}\end{array}\right)$.

We first estimate $\mathbb{E}\left[X_{2}\right]$. Since the hyperedges of $\left(\begin{array}{c}{[n]} \\ A P_{k}\end{array}\right)$ are arithmetic progressions of length $k$, every pair of vertices is contained in at most $\left(\begin{array}{l}k \\ 2\end{array}\right)$ such hyperedges. Consequently, we have

$$
\mathbb{E}\left[X_{2}\right] \leqslant\left(\begin{array}{l}
n \\
2
\end{array}\right)\left(\begin{array}{l}
k \\
2
\end{array}\right)^{2} p^{k+1} \leqslant k^{4} p^{k+1} n^{2}=p n \cdot \frac{k^{4} D_{p}^{k}}{n^{1 /(k-1)}}<\frac{t}{4}
$$


Next we bound $\mathbb{E}\left[X_{j}\right]$ for $3 \leqslant j<g$. For that we note that for any $j$-cycle we may first select and order the $j$ vertices of degree 2 and then fixing the remaining vertices of each edge. However, since every edge of the cycle contains two (already fixed) vertices of degree two, again there are at most $\left(\begin{array}{l}k \\ 2\end{array}\right)$ possible completion for such an edge and, hence, we have

$$
\sum_{j=3}^{g-1} \mathbb{E}\left[X_{j}\right] \leqslant \sum_{j=3}^{g-1} n^{j} k^{2 j} p^{(k-1) j}=\sum_{j=3}^{g-1} k^{2 j} D_{p}^{(k-1) j}<k^{2 g} D_{p}^{k g} \leqslant \frac{t}{4} .
$$

By Markov's inequality this implies that with probability less than $1 / 2$ the randomly generated hypergraph $\left(\begin{array}{l}{[n]_{p}} \\ A P_{k}\end{array}\right)$ has at most $t$ or more cycles of length less than $g$, which establishes Claim 4.1.

Proof of Claim 4.2. We consider the $k$-uniform hypergraph $\mathcal{H}=\left(\begin{array}{c}{[n]} \\ A P_{k}\end{array}\right)$ and check that it satisfies the assumptions of the Container Lemma (Theorem 2.1) for the parameters $\varepsilon$ and $\tau$ chosen in (13) and (17). Note that $\varepsilon<1 / 2$ by definition and $\tau<1 / 2$ follows from the choice of $n$ in (16). For the remaining assumption (1) we recall the definition of the average degrees $d_{j}$ for $j=1, \ldots, k$ of $\mathcal{H}$ and again, using the fact that every pair of vertices is contained in at most $\left(\begin{array}{l}k \\ 2\end{array}\right) A P_{k}$ 's, we note that for $j=2, \ldots, k$ we have

$$
d_{j} \leqslant d_{2} \leqslant\left(\begin{array}{l}
k \\
2
\end{array}\right)<\frac{k^{2}}{2} .
$$

Moreover, we have

$$
d_{1}=\frac{k}{n} \cdot\left|\left(\begin{array}{c}
{[n]} \\
A P_{k}
\end{array}\right)\right|=\frac{k}{n} \cdot \sum_{i=1}^{n-k+1}\left\lfloor\frac{n-i}{k-1}\right\rfloor=\frac{k}{n} \cdot \sum_{i=1}^{n-1}\left\lfloor\frac{n-i}{k-1}\right\rfloor \geqslant \frac{k}{n} \cdot \sum_{i=1}^{n-1}\left(\frac{n-i}{k-1}-1\right) \geqslant \frac{n}{2} .
$$

Consequently,

$$
\frac{6 \cdot k ! \cdot 2^{\left(\begin{array}{c}
k \\
2
\end{array}\right)}}{d_{1}} \sum_{j=2}^{k} \frac{d_{j}}{2^{\left(\begin{array}{c}
j-1 \\
2
\end{array}\right)} \tau^{j-1}} \leqslant \sum_{j=2}^{k} \frac{6 \cdot k ! \cdot 2^{\left(\begin{array}{c}
k \\
2
\end{array}\right)} \cdot k^{2}}{n \cdot 2^{\left(\begin{array}{c}
j-1 \\
2
\end{array}\right)} \cdot D_{\tau}^{j-1} n^{-\frac{j-1}{k-1}}}<\frac{6 \cdot k ! \cdot 2^{\left(\begin{array}{c}
k \\
2
\end{array}\right) \cdot k^{3}}}{\min \left\{D_{\tau}^{k-1}, n^{\frac{1}{k-1}}\right\}} \stackrel{(13),(16)}{\leqslant} \varepsilon .
$$

This shows that condition (1) of Theorem 2.1 holds. Consequently, for every independent set $I \subseteq V(\mathcal{H})$ we can apply conclusions $(i)-(i i i)$ of the Container Lemma with the constants defined in (14).

We consider the family $\mathcal{B}$ of all sets $B \subseteq[n]$ with the property that there exists a set $T \subseteq E(B)$ of size at most $t$ such that $(B \backslash T) \nrightarrow\left(A P_{k}\right)_{r}$, i.e., $\chi\left(\begin{array}{c}B \backslash T \\ A P_{k}\end{array}\right) \leqslant r$. Claim 4.2 is equivalent to

$$
\mathbb{P}\left([n]_{p} \in \mathcal{B}\right)<\frac{1}{2}
$$

By definition of $\mathcal{B}$, for every $B \in \mathcal{B}$ there exists a set $T^{B} \subseteq B$ of size $\left|T^{B}\right| \leqslant t$ and a partition $B=I_{1}^{B} \cup \ldots \cup I_{r}^{B} \cup T^{B}$ with the property that none of the sets $I_{i}^{B}$ contains an $A P_{k}$. In particular, each $I_{i}^{B}$ is an independent set in $\mathcal{H}$ and, therefore, properties $(i)-(i i i)$ of the 
Container Lemma assert that for every $i \in[r]$ there exists an $s$-tuple $\mathcal{S}_{i}^{B}=\left(\mathcal{S}_{i, 1}^{B}, \ldots, \mathcal{S}_{i, s}^{B}\right)$ of subsets of $I_{i}^{B}$ and a container set $\mathcal{C}\left(\mathcal{S}_{i}^{B}\right) \supseteq I_{i}^{B}$ such that

$$
\left|\mathcal{S}_{i, \sigma}^{B}\right| \leqslant \tau K n
$$

for every $\sigma \in[s]$ and

$$
\left|e_{\mathcal{H}}\left(\mathcal{C}\left(\mathcal{S}_{i}^{B}\right)\right)\right| \leqslant \varepsilon \cdot e(\mathcal{H})=\varepsilon\left|\left(\begin{array}{c}
{[n]} \\
A P_{k}
\end{array}\right)\right| .
$$

We set $\mathscr{S}^{B}=\left(\mathcal{S}_{1}^{B}, \ldots, \mathcal{S}_{r}^{B}\right)$ and $\mathscr{C}^{B}=\left(\mathcal{C}\left(\mathcal{S}_{1}^{B}\right), \ldots, \mathcal{C}\left(\mathcal{S}_{r}^{B}\right)\right)$.

Moreover, for any possible $r$-tuple $\mathscr{S}=\left(\mathcal{S}_{1}, \ldots, \mathcal{S}_{r}\right)$ of $s$-tuples of sets of size at most $\tau K n$, we consider the corresponding container vector $\mathscr{C}(\mathscr{S})=\left(\mathcal{C}\left(\mathcal{S}_{1}\right), \ldots, \mathcal{C}\left(\mathcal{S}_{r}\right)\right)$ given by the Container Lemma. We denote by $D(\mathscr{S})$ its complement in $[n]$ given by

$$
D(\mathscr{S})=[n] \backslash\left(\mathcal{C}\left(\mathcal{S}_{1}\right) \cup \cdots \cup \mathcal{C}\left(\mathcal{S}_{r}\right)\right) .
$$

Observe that for any $B \in \mathcal{B}$ the following two properties hold:

(a) $\bigcup_{i \in[r]} \bigcup_{\sigma \in[s]} \mathcal{S}_{i, \sigma}^{B} \subseteq\left(E(B) \backslash D\left(\mathscr{S}^{B}\right)\right)$ and

(b) $\left|B \cap D\left(\mathscr{S}^{B}\right)\right| \leqslant\left|T^{B}\right| \leqslant t$, since

$$
B \backslash T^{B}=I_{1}^{B} \cup \ldots \cup I_{r}^{B} \subseteq \mathcal{C}\left(\mathcal{S}_{1}^{B}\right) \cup \cdots \cup \mathcal{C}\left(\mathcal{S}_{r}^{B}\right)=[n] \backslash D\left(\mathscr{S}^{B}\right) .
$$

From $(a)$ and $(b)$ we infer that

$$
\begin{aligned}
\mathbb{P}\left([n]_{p} \in \mathcal{B}\right) & \leqslant \sum_{\mathscr{S}=\left(\mathcal{S}_{1}, \ldots, \mathcal{S}_{r}\right)} p\left|\cup_{i \in[r]} \cup_{\sigma \in[s]} \mathcal{S}_{i, \sigma}\right| \cdot \mathbb{P}\left(\left|[n]_{p} \cap D(\mathscr{S})\right| \leqslant t\right) \\
& \leqslant \max _{\mathscr{S}} \mathbb{P}\left(\left|[n]_{p} \cap D(\mathscr{S})\right| \leqslant t\right) \cdot \sum_{\mathscr{S}} p\left|\cup_{i \in[r]} \cup_{\sigma \in[s]} \mathcal{S}_{i, \sigma}\right|
\end{aligned}
$$

where the sum and the maximum are taken over all $r$-tuples $\mathscr{S}=\left(\mathcal{S}_{1}, \ldots, \mathcal{S}_{r}\right)$ of $s$-tuples $\mathcal{S}_{i}=\left(\mathcal{S}_{i, 1}, \ldots, \mathcal{S}_{i, s}\right)$ of sets $\mathcal{S}_{i, \sigma}$ of size at most $\tau K n$ for $i \in[r]$ and $\sigma \in[s]$. We will use property (ii) of the Container Lemma to bound the maximum probability in (21), while our choice of constants allow us to derive a sufficient bound for the sum in (21). We shall use the following fact (the proof of which we defer to the end of this section).

Fact 4.3. For every $(r+1)$-colouring of $[n]$ either there are more than $\left|\left(\begin{array}{c}{[n]} \\ A P_{k}\end{array}\right)\right| / W^{3}$ monochromatic $A P_{k}$ 's in the first $r$ colours or more than $\frac{n}{4 W}$ elements are in the last colour.

In view of this fact, we consider $\mathcal{C}\left(\mathcal{S}_{1}\right) \cup \cdots \cup \mathcal{C}\left(\mathcal{S}_{r}\right) \cup D(\mathscr{S})$ as an $(r+1)$-colouring of $[n]$. Owing to property (ii) of the Container Lemma (see (20)), every $\mathcal{C}\left(\boldsymbol{S}_{i}\right)$ contains at most $\varepsilon\left|\left(\begin{array}{c}{[n]} \\ A P_{k}\end{array}\right)\right|$ monochromatic $A P_{k}$ 's and, hence, there are at most

$$
r \cdot \varepsilon\left|\left(\begin{array}{c}
{[n]} \\
A P_{k}
\end{array}\right)\right| \stackrel{(13)}{=} \frac{1}{W^{3}}\left|\left(\begin{array}{c}
{[n]} \\
A P_{k}
\end{array}\right)\right|
$$


monochromatic $A P_{k}$ 's in the first $r$ colours. Therefore, Fact 4.3 yields that for every $\mathscr{S}=\left(\mathcal{S}_{1}, \ldots, \mathcal{S}_{r}\right)$ we have

$$
|D(\mathscr{S})|>\frac{n}{4 W}
$$

In particular, the choice of $t$ combined with (22) yields $t<p|D(\mathscr{S})| / 2$ and, consequently, Chernoff's inequality (see, e.g., [16, Theorem 2.1] asserts

$$
\mathbb{P}\left(\left|[n]_{p} \cap D(\mathscr{S})\right| \leqslant t\right) \leqslant \exp \left(-\frac{p n}{32 W}\right)
$$

for every $\mathscr{S}$ considered here. In particular, (23) bounds the maximum probability considered in the R-H-S of (21) and below we turn to the sum in (21).

Owing to $\left|\mathcal{S}_{i, \sigma}\right| \leqslant \tau K n$ for every $i \in[r]$ and $\sigma \in[s]$ we have

$$
\sum_{\mathscr{S}=\left(\mathcal{S}_{1}, \ldots, \mathcal{S}_{r}\right)} p^{\left|\bigcup_{i \in[r]} \bigcup_{\sigma \in[s]} \mathcal{S}_{i, \sigma}\right|} \leqslant \sum_{m=0}^{r \cdot s \cdot \tau K n}\left(\begin{array}{c}
n \\
m
\end{array}\right) 2^{r s m} p^{m} \leqslant \sum_{m=0}^{r \cdot s \cdot \tau K n}\left(\frac{\mathrm{e} n}{m} 2^{r s} p\right)^{m} .
$$

Since the function $m \mapsto\left(\mathrm{e} n 2^{r s} p / m\right)^{m}$ is unimodal and attains its maximum value for $m_{0}=2^{r s} p n \geqslant r s \tau K n$, from (24) we obtain

$$
\begin{aligned}
\sum_{\mathscr{S}=\left(\mathcal{S}_{1}, \ldots, \mathcal{S}_{r}\right)} p^{\left|\bigcup_{i \in[r]} \bigcup_{\sigma \in[s]} \mathcal{S}_{i, \sigma}\right|} & \leqslant(r s \tau K n+1) \cdot\left(\frac{\mathrm{e} n}{r s \tau K n} 2^{r s} p\right)^{r s \tau K n} \\
& \leqslant n \cdot\left(\frac{2^{r s} \mathrm{e} D_{p}}{r s K D_{\tau}}\right)^{r s \tau K n} \\
& \stackrel{(15)}{=} n \cdot\left(2^{r s+7} \mathrm{e} \cdot W r s \log (128 W r)\right)^{r s \tau K n} \\
& \leqslant n \cdot \exp (r s \tau K n(r s+6+\ln (W r s \log (128 W r)))) \\
& \stackrel{(15)}{\leqslant} n \cdot \exp \left(\frac{p n}{128 W}\right) \\
& \leqslant \exp \left(\frac{p n}{64 W}\right) .
\end{aligned}
$$

Finally, combining (23) and (25) with (21) leads to

$$
\mathbb{P}\left([n]_{p} \in \mathcal{B}\right) \leqslant \exp \left(-\frac{p n}{32 W}\right) \cdot \exp \left(\frac{p n}{64 W}\right)=\exp \left(-\frac{p n}{64 W}\right)<\frac{1}{2} .
$$

Up to the proof of Fact 4.3 this concludes the proof of Claim 4.2.

Proof of Fact 4.3. Recall that $W=\operatorname{vdW}(k ; r)$ asserts that $A \rightarrow\left(A P_{k}\right)_{r}$ for every arithmetic progression $A \subseteq \mathbb{N}$ of length $W$. Consider an arbitrary $(r+1)$-colouring of $[n]$. Suppose at most $\frac{n}{4 W}$ elements of $[n]$ receive colour $r+1$. From the observation that for every $w \leqslant W / 2$ and every $i \in[n]$ there are at most $\frac{n-1}{W-w} \operatorname{distinct} A P_{W}$ 's in $[n]$ having $i$ 
at position $w$ or $W-w+1$, one can deduce that every $i \in[n]$ is contained in at most $n$ different $A P_{W}$ 's. Consequently, there are at least

$$
\left|\left(\begin{array}{c}
{[n]} \\
A P_{W}
\end{array}\right)\right|-\frac{n^{2}}{4 W} \geqslant \frac{n^{2}}{4 W}
$$

$A P_{W}$ 's containing no element of the last colour, where we used $\left|\left(\begin{array}{c}{[n]} \\ A P_{W}\end{array}\right)\right| \geqslant \frac{n^{2}}{2 W}$ for the last inequality (cf. (18)).

Owing to the choice of $W$ every such $r$-coloured $A P_{W}$ contains a monochromatic $A P_{k}$ in one of the first $r$ colours. On the other hand, every $A P_{k}$ can be contained in at most $\left(\begin{array}{c}W \\ 2\end{array}\right)$ different $A P_{W}$ 's in $[n]$. Therefore, there exist at least

$$
\frac{n^{2}}{4 W} \cdot \frac{2}{W^{2}}=\frac{n^{2}}{2 W^{3}} \geqslant \frac{1}{W^{3}} \cdot\left|\left(\begin{array}{c}
{[n]} \\
A P_{k}
\end{array}\right)\right|
$$

distinct monochromatic $A P_{k}$ 's in $[n]$ coloured in one of the first $r$ colours and the fact follows.

\section{§5. Proof of Theorem 1.4}

In this section we establish that for all integers $r \geqslant 2, g \geqslant 3$, and $k \geqslant 3$, there exists a graph $H$ on at most $k^{40 g k^{4}} R^{40 g k^{2}}$ vertices such that $\left(\begin{array}{c}H \\ K_{k}\end{array}\right)$ has chromatic number greater than $r$ and girth at least $g$, where $R=R\left(K_{k} ; r\right)$ is the $r$-colour Ramsey number for $K_{k}$.

Proof. We first define all constants involved in the proof. Given the uniformity $k \geqslant 3$, the number of colours $r \geqslant 2$, and the minimum girth $g \geqslant 3$, we denote by $R=R\left(K_{k} ; r\right)$ the $r$-colour Ramsey number for $K_{k}$. In the estimates below we sometimes use the trivial observation $2^{r}<R$. For a later application of the Container Lemma (Theorem 2.1), we define the involved auxiliary constants (and observe some immediate bounds)

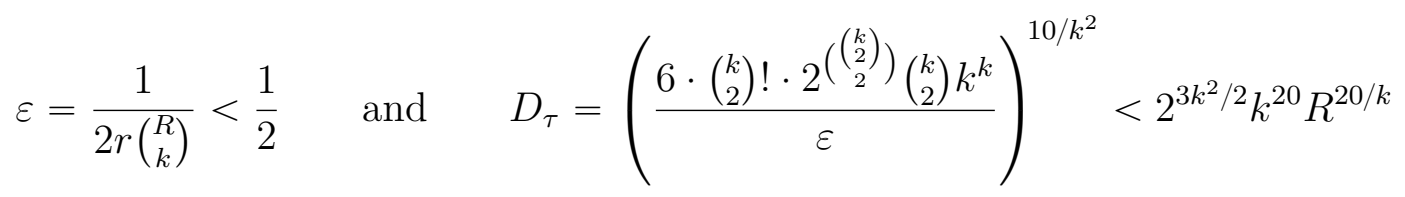

and integers

$$
K=800\left(\begin{array}{c}
k \\
2
\end{array}\right)\left(\left(\begin{array}{c}
k \\
2
\end{array}\right) !\right)^{3}<k^{3 k^{2}} \quad \text { and } \quad s=\lfloor K \log (1 / \varepsilon)\rfloor<k^{3 k^{2}} \log \left(r R^{k}\right) .
$$

We introduce another auxiliary constant

$$
D_{p}=50 R^{2} r^{2} s^{2} K D_{\tau} \log \left(50 R^{2} r\right)<k^{10 k^{2}+30} R^{5+20 / k}
$$

and set

$$
n=D_{p}^{k^{2}(5+g)}<D_{p}^{3 k^{2} g}<k^{40 g k^{4}} R^{40 g k^{2}} .
$$


Finally, we define the following three parameters in terms of some of the constants above

$$
\tau=\frac{D_{\tau}}{n^{2 /(k+1)}}, \quad p=\frac{D_{p}}{n^{2 /(k+1)}}, \quad \text { and } \quad t=\frac{p}{2 R^{2}}\left(\begin{array}{l}
n \\
2
\end{array}\right) .
$$

Having defined all involved constants we shall show the following two claims, which yield the theorem.

Claim 5.1. With probability larger than $1 / 2$, the random graph $G(n, p)$ has the property that there is a set $T \subseteq E(G(n, p))$ of size at most t such that $\operatorname{girth}\left(\begin{array}{c}G(n, p)-T \\ K_{k}\end{array}\right) \geqslant g$.

Claim 5.2. With probability larger than $1 / 2$, the random graph $G(n, p)$ has the property that $\chi\left(\begin{array}{c}G(n, p)-T \\ K_{k}\end{array}\right)>r$ for every subset $T \subseteq E(G(n, p))$ of size at most $t$.

Both claims together show that with positive probability there exists a graph $G$ which contains a set $T \subseteq E(G)$ of size at most $t$ such that $H=G-T$ satisfies girth $\left(\begin{array}{c}H \\ K_{k}\end{array}\right) \geqslant g$ and $\chi\left(\begin{array}{c}H \\ K_{k}\end{array}\right)>r$. Consequently, Theorem 1.4 follows from the choice of $n$ in (29).

Proof of Claim 5.1. Recall that a 2-cycle is a pair of edges $e_{1}, e_{2}$ such that $\left|e_{1} \cap e_{2}\right|>1$ and for $j>2$ a $j$-cycle is a cyclical sequence of $j$ edges $e_{1}, e_{2}, \ldots, e_{j}$ where the intersection of two consecutive edges is exactly one i.e. $\left|e_{i} \cap e_{i+1}\right|=1$ (addition mod $j$ ), the intersection of any two nonconsecutive edges is empty, and the intersection points for each pair of consecutive edges is unique.

Define $X_{j}$ to be the number of $j$-cycles in the system of copies of $K_{k}$ in $G(n, p)$. We first work to bound $X_{2}$. If $k=3$, we trivially have $\mathbb{E}\left[X_{2}\right]=0$. Otherwise for $k \geqslant 4$, a 2-cycle corresponds to two copies of $K_{k}$ that intersect in more than two edges, and thus in more than two vertices. Furthermore, we see that two copies of $K_{k}$ that intersect in $i$ vertices together span exactly $2 k-i$ vertices and $2\left(\begin{array}{l}k \\ 2\end{array}\right)-\left(\begin{array}{l}i \\ 2\end{array}\right)$ edges. With this in mind, the following bounds $\mathbb{E}\left[X_{2}\right]<t / 4$ in $\left(\begin{array}{c}G(n, p) \\ K_{k}\end{array}\right)$ :

$$
\begin{aligned}
& \frac{\mathbb{E}\left[X_{2}\right]}{t / 4}=\frac{8 R^{2}}{p\left(\begin{array}{l}
n \\
2
\end{array}\right)} \cdot \mathbb{E}\left[X_{2}\right] \leqslant \frac{32 R^{2}}{p n^{2}} \cdot \sum_{i=3}^{k-1} n^{2 k-i} p^{2}\left(\begin{array}{c}
k \\
2
\end{array}\right)-\left(\begin{array}{c}
i \\
2
\end{array}\right) \\
& =32 R^{2} n^{2 k-2} p^{2\left(\begin{array}{c}
k \\
2
\end{array}\right)-1} \sum_{i=3}^{k-1} n^{\left(i^{2}-2 i-k i\right) /(k+1)} D_{p}^{-\left(\begin{array}{c}
i \\
2
\end{array}\right)} \\
& \leqslant 32 R^{2} n^{2 k-2} p^{2\left(\begin{array}{c}
k \\
2
\end{array}\right)-1} \cdot k \cdot \max _{3 \leqslant i \leqslant k-1}\left\{n^{\left(i^{2}-2 i-k i\right) /(k+1)}\right\} \\
& \leqslant 32 R^{2} n^{2 k-2} p^{2\left(\begin{array}{c}
k \\
2
\end{array}\right)-1} \cdot k \cdot n^{(3-3 k) /(k+1)} \\
& =\frac{32 k R^{2} D_{p}^{k^{2}-k-1}}{n^{(k-3) /(k+1)}} \leqslant \frac{D_{p}^{k^{2}}}{n^{1 / 5}} \stackrel{(29)}{<} 1 .
\end{aligned}
$$


We now bound $\sum_{j=3}^{g-1} X_{j}$. For $j>2$, a $j$-cycle in $\left(\begin{array}{c}K_{n} \\ K_{k}\end{array}\right)$ consists of a cyclically ordered set of $j$ copies of $K_{k}$ such that each two consecutive copies intersect in exactly one edge of $K_{n}$. Thus, a $j$-cycle corresponds to a set of $K_{k}$ 's in $K_{n}$ that span at most $k j-2 j$ vertices in $K_{n}$ and exactly $\left(\begin{array}{l}k \\ 2\end{array}\right) j-j$ edges in $K_{n}$. From this, we see that, for $2<j<g$, we have

$$
\mathbb{E}\left[X_{j}\right] \leqslant n^{k j-2 j} p^{\left(\begin{array}{c}
k \\
2
\end{array}\right) j-j}=\left(n^{k-2} p^{\left(\begin{array}{c}
k \\
2
\end{array}\right)-1}\right)^{j}=D_{p}^{\left.\left(\begin{array}{l}
k \\
2
\end{array}\right)-1\right) j} .
$$

Using this, we establish $\sum_{j=3}^{g-1} \mathbb{E}\left[X_{j}\right]<t / 4$ :

$$
\frac{\sum_{j=3}^{g-1} \mathbb{E}\left[X_{j}\right]}{t / 4} \leqslant \frac{8 R^{2}}{p\left(\begin{array}{c}
n \\
2
\end{array}\right)} \cdot g \cdot D_{p}^{\left(\left(\begin{array}{c}
k \\
2
\end{array}\right)-1\right) g} \leqslant \frac{32 R^{2} g}{p n^{2}} D_{p}^{\left(\left(\begin{array}{c}
k \\
2
\end{array}\right)-1\right) g} \leqslant \frac{D_{p}^{k^{2} g}}{n}<1 .
$$

Thus, we have shown $\sum_{j=2}^{g-1} \mathbb{E}\left[X_{j}\right]<t / 4+t / 4=t / 2$. By Markov's inequality, this gives that, with probability bigger $1 / 2$, the hypergraph $\left(\begin{array}{c}G(n, p) \\ K_{k}\end{array}\right)$ contains less than $t$ cycles of length less than $g$. For each such cycle, removing one vertex (which is an edge in $G(n, p)$ ) concludes the proof of Claim 5.1.

Proof of Claim 5.2. The proof relies on an application of the Container Lemma (Theorem 2.1) to the $\left(\begin{array}{l}k \\ 2\end{array}\right)$-uniform hypergraph $\mathcal{H}=\left(\begin{array}{c}K_{n} \\ K_{k}\end{array}\right)$ (for similar proofs see, e.g., [17, 24]). In view of that we will first verify condition (1) for our choices of $\varepsilon$ and $\tau$ in (26) and (30). Recalling the definition of the average degrees $d_{j}$ for $j=1, \ldots,\left(\begin{array}{l}k \\ 2\end{array}\right)$ of $\mathcal{H}$, we note that

$$
d_{1}=\left(\begin{array}{l}
n-2 \\
k-2
\end{array}\right) \geqslant \frac{n^{k-2}}{k^{k}} .
$$

For $j \geqslant 2$, letting $k_{j}$ be the smallest integer such that $j \leqslant\left(\begin{array}{c}k_{j} \\ 2\end{array}\right)$, we have

$$
d_{j} \leqslant\left(\begin{array}{c}
n-k_{j} \\
k-k_{j}
\end{array}\right) \leqslant n^{k-k_{j}} .
$$

Consequently, for every $j=2, \ldots,\left(\begin{array}{l}k \\ 2\end{array}\right)$ this gives

$$
\frac{d_{j}}{d_{1} \cdot \tau^{j-1}} \leqslant \frac{k^{k} \cdot n^{2-k_{j}} \cdot n^{\frac{2 j-2}{k+1}}}{D_{\tau}^{j-1}} \leqslant \frac{k^{k} \cdot n^{2-k_{j}} \cdot n^{\frac{2\left(\begin{array}{l}
k_{j} \\
2
\end{array}\right)-2}{k+1}}}{D_{\tau}^{j-1}}=\frac{k^{k} \cdot n^{\frac{\left(k_{j}-2\right)\left(k_{j}-k\right)}{k+1}}}{D_{\tau}^{j-1}} .
$$

For $k_{j}=k$, i.e., for $j=\left(\begin{array}{c}k-1 \\ 2\end{array}\right)+1, \ldots,\left(\begin{array}{c}k \\ 2\end{array}\right)$ we, therefore, get

$$
\frac{d_{j}}{d_{1} \cdot \tau^{j-1}} \leqslant \frac{k^{k}}{D_{\tau}^{j-1}} \leqslant \frac{k^{k}}{D_{\tau}^{\left(\begin{array}{c}
(k-1) \\
2
\end{array}\right.}} \leqslant \frac{k^{k}}{D_{\tau}^{k^{2} / 10}}
$$

where we used $D_{\tau} \geqslant 1$ and $k \geqslant 3$ for the last inequalities. For integers $3 \leqslant k_{j} \leqslant k-1$ we note that $k \geqslant 4$ and $\left(k_{j}-2\right)\left(k_{j}-k\right)$ is maximized for $k_{j}=3$ (and $k_{j}=k-1$ ). Hence, in this case we can bound the R-H-S in (31) to give

$$
\frac{d_{j}}{d_{1} \cdot \tau^{j-1}} \leqslant \frac{k^{k}}{n^{1 / 5}} .
$$


Summarizing, since $k_{j} \geqslant 3$ for $j \geqslant 2$ we arrive for $h=\left(\begin{array}{l}k \\ 2\end{array}\right)$ at

$$
\frac{6 \cdot h ! \cdot 2^{\left(\begin{array}{c}
h \\
2
\end{array}\right)}}{d_{1}} \sum_{j=2}^{h} \frac{d_{j}}{2^{\left(\begin{array}{c}
j-1 \\
2
\end{array}\right)} \tau^{j-1}} \leqslant \frac{6 \cdot h ! \cdot 2^{\left(\begin{array}{c}
h \\
2
\end{array}\right)} \cdot h \cdot k^{k}}{\min \left\{D_{\tau}^{k^{2} / 10}, n^{1 / 5}\right\}} \stackrel{(26),(29)}{\leqslant} \varepsilon
$$

and this shows that condition (1) of Theorem 2.1 holds. Consequently, for every independent set $I \subseteq V(\mathcal{H})$ we can apply conclusions $(i)-(i i i)$ of the Container Lemma with the constants defined above.

We consider the family $\mathcal{B}$ of all graphs $B \subseteq K_{n}$ such that there exists a set $T \subseteq E(B)$ of size at most $t$ and $(B-T) \nrightarrow\left(K_{k}\right)_{r}$, i.e., there exists and $r$-colouring of the edges of the graph $B-T$ without a monochromatic copy of $K_{k}$. In other words, $\chi\left(\begin{array}{c}B-T \\ K_{k}\end{array}\right) \leqslant r$ and we may view $\mathcal{B}$ as the set of all ('bad') graphs on $n$ vertices that do not have the desired property of Claim 5.2. Below we establish Claim 5.2 by showing

$$
\mathbb{P}(G(n, p) \in \mathcal{B})<\frac{1}{2}
$$

Consider any graph $B \in \mathcal{B}$. By the definition of $\mathcal{B}$, there exists a set $T^{B} \subseteq E(B)$ of size $\left|T^{B}\right| \leqslant t$ and a partition $E(B) \backslash T^{B}=I_{1}^{B} \cup \ldots \cup I_{r}^{B}$ with the property that none of the sets $I_{i}^{B}$ contains a $K_{k}$. In particular, each $I_{i}^{B}$ is an independent set in $\mathcal{H}$ and, therefore, properties $(i)-(i i i)$ of the Container Lemma assert that for every $i \in[r]$ there exists an $s$-tuple $\mathcal{S}_{i}^{B}=\left(\mathcal{S}_{i, 1}^{B}, \ldots, \mathcal{S}_{i, s}^{B}\right)$ of subsets of $I_{i}^{B}$ and a container set $\mathcal{C}\left(\mathcal{S}_{i}^{B}\right) \supseteq I_{i}^{B}$ such that

$$
\left|\mathcal{S}_{i, \sigma}^{B}\right| \leqslant \tau K\left(\begin{array}{l}
n \\
2
\end{array}\right)
$$

for every $\sigma \in[s]$ and

$$
\left|e_{\mathcal{H}}\left(\mathcal{C}\left(\mathcal{S}_{i}^{B}\right)\right)\right| \leqslant \varepsilon\left(\begin{array}{l}
n \\
k
\end{array}\right)
$$

We also set $\mathscr{S}^{B}=\left(\mathcal{S}_{1}^{B}, \ldots, \mathcal{S}_{r}^{B}\right)$ and $\mathscr{C}^{B}=\left(\mathcal{C}\left(\mathcal{S}_{1}^{B}\right), \ldots, \mathcal{C}\left(\mathcal{S}_{r}^{B}\right)\right)$.

Moreover, for any possible $r$-tuple $\mathscr{S}=\left(\mathcal{S}_{1}, \ldots, \mathcal{S}_{r}\right)$ of $s$-tuples of sets of size at most $\tau K\left(\begin{array}{l}n \\ 2\end{array}\right)$ we consider the corresponding container vector $\mathscr{C}(\mathscr{S})=\left(\mathcal{C}\left(\mathcal{S}_{1}\right), \ldots, \mathcal{C}\left(\mathcal{S}_{r}\right)\right)$ given by the Container Lemma. We denote by $D(\mathscr{S})$ its complement in $E\left(K_{n}\right)$ given by

$$
D(\mathscr{S})=E\left(K_{n}\right) \backslash\left(\mathcal{C}\left(\mathcal{S}_{1}\right) \cup \cdots \cup \mathcal{C}\left(\mathcal{S}_{r}\right)\right)
$$

We observe that for any $B \in \mathcal{B}$ the following two properties hold:

(a) $\bigcup_{i \in[r]} \bigcup_{\sigma \in[s]} \mathcal{S}_{i, \sigma}^{B} \subseteq\left(E(B) \backslash D\left(\mathscr{S}^{B}\right)\right)$ and

(b) $\left|E(B) \cap D\left(\mathscr{S}^{B}\right)\right| \leqslant\left|T^{B}\right| \leqslant t$, since

$$
E(B) \backslash T^{B}=I_{1}^{B} \cup \ldots \cup I_{r}^{B} \subseteq \mathcal{C}\left(\mathcal{S}_{1}^{B}\right) \cup \cdots \cup \mathcal{C}\left(\mathcal{S}_{r}^{B}\right)=E\left(K_{n}\right) \backslash D\left(\mathscr{S}^{B}\right)
$$


From $(a)$ and $(b)$ we infer that

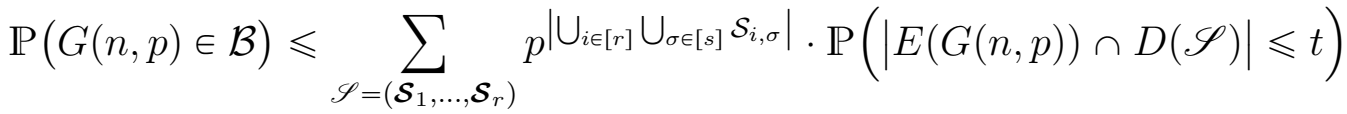

$$
\begin{aligned}
& \leqslant \max _{\mathscr{S}} \mathbb{P}(|E(G(n, p)) \cap D(\mathscr{S})| \leqslant t) \sum_{\mathscr{S}} p\left|\bigcup_{i \in[r]} \bigcup_{\sigma \in[s]} \mathcal{S}_{i, \sigma}\right|,
\end{aligned}
$$

where the sum and the maximum are taken over all $r$-tuples $\mathscr{S}=\left(\mathcal{S}_{1}, \ldots, \mathcal{S}_{r}\right)$ of $s$-tuples $\mathcal{S}_{i}=\left(\mathcal{S}_{i, 1}, \ldots, \mathcal{S}_{i, s}\right)$ of sets $\mathcal{S}_{i, \sigma}$ of size at most $\tau K\left(\begin{array}{l}n \\ 2\end{array}\right)$ for $i \in[r]$ and $\sigma \in[s]$. We will use property $(i i)$ of the Container Lemma to bound the maximum probability in (35), while our choice of constants allow us to derive a sufficient bound for the sum in (35).

For the maximum probability we first observe that for every $\mathscr{S}=\left(\mathcal{S}_{1}, \ldots, \mathcal{S}_{r}\right)$ we have

$$
|D(\mathscr{S})|>\frac{1}{R^{2}}\left(\begin{array}{l}
n \\
2
\end{array}\right) .
$$

For the proof we use the fact that for any $(r+1)$-colouring of $E\left(K_{n}\right)$ either there are more than

$$
\frac{1}{2\left(\begin{array}{l}
R \\
k
\end{array}\right)}\left(\begin{array}{l}
n \\
k
\end{array}\right)
$$

monochromatic copies of $K_{k}$ in the first $r$ colours or there are more than $\frac{1}{R^{2}}\left(\begin{array}{l}n \\ 2\end{array}\right)$ edges having the last colour (see, e.g., [24, Proposition 8]).

In view of this fact, we consider $\mathcal{C}\left(\mathcal{S}_{1}\right) \cup \cdots \cup \mathcal{C}\left(\boldsymbol{S}_{r}\right) \cup D(\mathscr{S})$ as an $(r+1)$-colouring of $E\left(K_{n}\right)$. Owing to property (ii) of the Container Lemma (see (34)), every $\mathcal{C}\left(\boldsymbol{S}_{i}\right)$ contains at most $\varepsilon\left(\begin{array}{l}n \\ k\end{array}\right)$ copies of $K_{k}$ and, hence, there are at most

$$
r \cdot \varepsilon\left(\begin{array}{l}
n \\
k
\end{array}\right) \stackrel{(26)}{=} \frac{1}{2\left(\begin{array}{l}
R \\
k
\end{array}\right)}\left(\begin{array}{l}
n \\
k
\end{array}\right)
$$

monochromatic copies in the first $r$ colours. Therefore, the mentioned fact above yields (36). In particular, the choice of $t$ combined with (36) yields $t<p|D(\mathscr{S})| / 2$ and, consequently, Chernoff's inequality (see, e.g., [16, Theorem 2.1]) asserts

$$
\mathbb{P}(|E(G(n, p)) \cap D(\mathscr{S})| \leqslant t) \leqslant \exp \left(-\frac{p}{8 R^{2}}\left(\begin{array}{l}
n \\
2
\end{array}\right)\right)
$$

for every $\mathscr{S}$ considered here. In particular, (37) bounds the maximum probability considered in the R-H-S of (35) and below we turn to the sum in (35).

Owing to $\left|\mathcal{S}_{i, \sigma}\right| \leqslant \tau K\left(\begin{array}{l}n \\ 2\end{array}\right)$ for every $i \in[r]$ and $\sigma \in[s]$ we have

$$
\left.\sum_{\mathscr{S}=\left(\mathcal{S}_{1}, \ldots, \mathcal{S}_{r}\right)} p^{\left|\bigcup_{i \in[r]} \bigcup_{\sigma \in[s]} \mathcal{S}_{i, \sigma}\right|} \leqslant \sum_{m=0}^{r \cdot s \cdot \tau K\left(\begin{array}{c}
n \\
2
\end{array}\right)}\left(\begin{array}{c}
n \\
2
\end{array}\right)\right) 2^{r s m} p^{m} \leqslant \sum_{m=0}^{r \cdot s \cdot \tau K\left(\begin{array}{c}
n \\
2
\end{array}\right)}\left(\frac{\mathrm{e}\left(\begin{array}{c}
n \\
2
\end{array}\right)}{m} 2^{r s} p\right)^{m} .
$$


Since the function $m \mapsto\left(\mathrm{e}\left(\begin{array}{l}n \\ 2\end{array}\right) 2^{r s} p / m\right)^{m}$ is unimodal and attains its maximum value for $m_{0}=\left(\begin{array}{l}n \\ 2\end{array}\right) 2^{r s} p \geqslant \operatorname{rs\tau } K\left(\begin{array}{l}n \\ 2\end{array}\right)$ (see (28)), we can bound the summands in R-H-S above by the last one and obtain

$$
\begin{aligned}
\sum_{\mathscr{S}=\left(\mathcal{S}_{1}, \ldots, \mathcal{S}_{r}\right)} p^{\left|\bigcup_{i \in[r]} \bigcup_{\sigma \in[s]} \mathcal{S}_{i, \sigma}\right|} & \leqslant\left(r s \tau K\left(\begin{array}{l}
n \\
2
\end{array}\right)+1\right) \cdot\left(\frac{\mathrm{e}\left(\begin{array}{l}
n \\
2
\end{array}\right)}{r s \tau K\left(\begin{array}{l}
n \\
2
\end{array}\right)} 2^{r s} p\right)^{r s \tau K\left(\begin{array}{l}
n \\
2
\end{array}\right)} \\
& \leqslant n^{2} \cdot\left(\frac{2^{r s} D_{p}}{r s K D_{\tau}}\right)^{r s \tau K\left(\begin{array}{l}
n \\
2
\end{array}\right)} \\
& \stackrel{(28)}{=} n^{2} \cdot\left(2^{r s} \mathrm{e} \cdot 50 R^{2} r s \log \left(50 R^{2} r\right)\right)^{r s \tau K\left(\begin{array}{l}
n \\
2
\end{array}\right)} \\
& \leqslant n^{2} \cdot \exp \left(r s \tau K\left(\begin{array}{l}
n \\
2
\end{array}\right)\left(r s+1+\ln \left(50 R^{2} r s \log \left(50 R^{2} r\right)\right)\right)\right) \\
& \stackrel{(28)}{\leqslant} n^{2} \cdot \exp \left(\frac{p}{16 R^{2}}\left(\begin{array}{l}
n \\
2
\end{array}\right)\right) \\
& \leqslant \exp \left(\frac{p}{12 R^{2}}\left(\begin{array}{l}
n \\
2
\end{array}\right)\right) .
\end{aligned}
$$

Finally, combining (37) and (38) with (35) leads to

$$
\mathbb{P}(G(n, p) \in \mathcal{B}) \leqslant \exp \left(-\frac{p}{8 R^{2}}\left(\begin{array}{l}
n \\
2
\end{array}\right)\right) \cdot \exp \left(\frac{p}{12 R^{2}}\left(\begin{array}{l}
n \\
2
\end{array}\right)\right)=\exp \left(-\frac{p}{24 R^{2}}\left(\begin{array}{l}
n \\
2
\end{array}\right)\right)<\frac{1}{2}
$$

and Claim 5.2 follows.

\section{§6. Concluding Remarks}

In view of Theorem 1.1 we may consider the following function for given integers $r \geqslant 2$ and $k \geqslant 3$

$$
f_{r}(k)=\min \left\{|V(H)|: \operatorname{girth}(H)=k \text { and } H \rightarrow\left(C_{k}\right)_{r}\right\} .
$$

Theorem 1.1 established that $f_{r}(k) \leqslant R^{10 k^{2}} k^{15 k^{3}}$, where $R=R\left(C_{k} ; r\right)$. In view of the known upper bounds on $R\left(C_{k} ; r\right)$ for even and odd $k$, this establishes the upper bounds stated below in Theorems 6.1 and 6.2. These two theorems also provide complementary lower bounds.

Theorem 6.1. There exist positive constants $c_{1}$ and $c_{2}$ such that for all $k \geqslant 2$ and $r \geqslant 2$,

$$
\exp \left(c_{1} k \log r\right) \leqslant f_{r}(2 k) \leqslant \exp \left(c_{2}\left(k^{2} \log r+k^{3} \log k\right)\right) .
$$

For fixed $k \geqslant 2$ Theorem 6.1 shows that $f_{r}(2 k)$ is polynomial in $r$.

Proof. We will first show that $f_{r}(2 k) \leqslant \exp \left(c_{2}\left(k^{2} \log r+k^{3} \log k\right)\right)$. In [7] it was announced and in [5] it was proved that, for every integer $k \geqslant 2$, there exists a constant $c$ such that every graph on $n$ vertices with at least $c n^{1+1 / k}$ edges contains a copy of the cycle $C_{2 k}$. This 
implies that, if $n$ is such that $\left(\begin{array}{l}n \\ 2\end{array}\right) / r \geqslant c n^{1+1 / k}$, i.e. $n \geqslant c r^{k /(k-1)}$, then every edge colouring of $K_{n}$ with $r$ colours will have a monochromatic cycle $C_{2 k}$. Hence

$$
R\left(C_{2 k} ; r\right) \leqslant c r^{k /(k-1)} .
$$

The upper bound $f_{r}(2 k) \leqslant \exp \left(c_{2}\left(k^{2} \log r+k^{3} \log k\right)\right)$ now follows from substituting (39) into Theorem 1.1.

We now turn our attention towards the lower bound in Theorem 6.1. For any $k \geqslant 2$ and $r \geqslant 2$ consider any graph $H$ with girth $(H)=2 k$ and the property $H \rightarrow\left(C_{2 k}\right)_{r}$. Let $\tilde{H} \subseteq H$ be an edge minimal subgraph such that $\widetilde{H} \rightarrow\left(C_{2 k}\right)_{r}$. Clearly the minimum degree of $\widetilde{H}$ must be at least $r$ and $\widetilde{H}$ must have girth at least $2 k$. Since any graph with girth $2 k$ and minimum degree $r$ must have at least $2 \sum_{i=0}^{k-1}(r-1)^{i} \geqslant c r^{k-1}$ vertices the lower bound for $f_{r}(2 k)$ follows.

The following theorem establishes a similar results for the odd case.

Theorem 6.2. There exist positive constants $c_{1}$ and $c_{2}$ such that, for all $k \geqslant 1$ and $r \geqslant 2$,

$$
\exp \left(c_{1} k r\right) \leqslant f_{r}(2 k+1) \leqslant \exp \left(c_{2} k^{2}(r \log r+k \log k)\right)
$$

For fixed $k \geqslant 2$ it follows that $e^{\Omega(r)} \leqslant f_{r}(2 k+1) \leqslant e^{O(r \log r)}$.

Proof. We fist show that $f_{r}(2 k+1) \leqslant \exp \left(c_{2} k^{2}(r \log r+k \log k)\right)$. As established in [4],

$$
2^{r} k \leqslant R\left(C_{2 k+1} ; r\right) \leqslant(r+2) ! \cdot(2 k+1) .
$$

The upper bound for $f_{r}(2 k+1)$ follows from substituting the upper bound in (40) into Theorem 1.1.

To establish that $f_{r}(2 k+1) \geqslant \exp \left(c_{1} k r\right)$ for any $k \geqslant 1$ and $r \geqslant 2$, as before we begin by considering any graph $H$ with $\operatorname{girth}(H)=2 k+1$ and the property $H \rightarrow\left(C_{2 k+1}\right)_{r}$. Note that $\chi(H)>2^{r}$, since otherwise the edges of $H$ could be decomposed into $r$ bipartite graphs, resulting in an $r$-colouring of $E(H)$ with no monochromatic odd cycle. Moreover, since $\chi(H)>2^{r}$, there must be a subgraph $\widetilde{H} \subset H$ with minimum degree at least $2^{r}$. Since $\tilde{H}$ has at least girth $2 k+1$ and minimum degree $2^{r}$, the number of vertices in $\tilde{H}$ must be at least $1+2^{r} \sum_{i=1}^{k-1}\left(2^{r}-1\right)^{i} \geqslant 2^{\text {crk }}$ vertices for some $c>0$.

For three special cases of $k$, we are able to deduce better bounds for $f_{r}(2 k)$ using well known extremal constructions of graphs with girth 6,8 , and 12 , respectively.

Theorem 6.3. We have $f_{r}(6)=O\left(r^{6}\right), f_{r}(8)=O\left(r^{12}\right)$, and $f_{r}(12)=O\left(r^{30}\right)$.

Before proving Theorem 6.3, we first introduce some notation and state an observation upon which the proof if based. Let $\operatorname{ex}\left(n ; C_{k}\right)$ denote the maximum number of edges in an $n$ 
vertex graph that does not contain a cycle of length $k$. Similarly, let $\operatorname{ex}\left(n ; C_{3}, C_{4}, \ldots, C_{k-1}\right)$ denote the maximum number of edges in a graph with girth $k$.

Fact 6.4. If $\operatorname{ex}\left(n ; C_{3}, C_{4}, \ldots, C_{2 k-1}\right)>r \cdot \operatorname{ex}\left(n ; C_{3}, C_{4}, \ldots, C_{2 k}\right)$, then $f_{r}(2 k) \leqslant n$.

Indeed, by definition of the extremal function there exists a graph $G$ on $n$ vertices with girth $2 k$ that has $\operatorname{ex}\left(n ; C_{3}, C_{4}, \ldots, C_{2 k-1}\right)$ edges. Clearly, every $r$-colouring of $G$ yields a monochromatic subgraph with at least $\operatorname{ex}\left(n ; C_{3}, C_{4}, \ldots, C_{2 k-1}\right) / r>\operatorname{ex}\left(n ; C_{3}, C_{4}, \ldots, C_{2 k}\right)$ edges, which must contain a monochromatic $C_{2 k}$ since the monochromatic subgraph still has girth at least $2 k$.

Proof of Theorem 6.3. To make use of this fact to prove Theorem 6.3, we use the result of Erdős and Simonovits from [13] that for every positive integer $k$, we have

$$
\operatorname{ex}\left(n ; C_{3}, C_{4}, \ldots, C_{2 k+1}\right)=O\left(n^{1+1 / k}\right)
$$

Since any graph contains a bipartite subgraph with half of its edges we have

$$
\begin{aligned}
\operatorname{ex}\left(n ; C_{3}, C_{4}, C_{5}, C_{6}, \ldots, C_{2 k}\right) & \leqslant \operatorname{ex}\left(n ; C_{4}, C_{6}, \ldots, C_{2 k}\right) \\
& \leqslant 2 \cdot \operatorname{ex}\left(n ; C_{3}, C_{4}, C_{5}, C_{6}, \ldots, C_{2 k+1}\right)=O\left(n^{1+1 / k}\right) .
\end{aligned}
$$

Erdős and Simonovits conjectured in [13] that for every positive integer $k \geqslant 2$,

$$
\operatorname{ex}\left(n ; C_{3}, C_{4}, \ldots, C_{2 k-1}\right)=\Omega\left(n^{1+1 /(k-1)}\right)
$$

This has been observed for $k=3$ by Klein (see [6]) and follows for $k=4$ by the work of Singleton [27], and for $k=6$ by the work of Benson [2]. For $k \in\{3,4,6\}$, inequalities (41) and (42) give that

$$
\operatorname{ex}\left(n ; C_{3}, C_{4}, \ldots, C_{2 k-1}\right)=\Omega\left(n^{1+1 /(k-1)}\right)>r \cdot O\left(n^{1+1 / k}\right)=r \cdot \operatorname{ex}\left(n ; C_{3}, C_{4}, \ldots, C_{2 k}\right)
$$

holds, provided that

$$
n \geqslant \tilde{c} r^{k(k-1)}
$$

for some sufficiently large constant $\tilde{c}$. Consequently, Fact 6.4 yields $f_{r}(2 k) \leqslant n=\Omega\left(r^{k(k-1)}\right)$ for $k \in\{3,4,6\}$ and the theorem follows.

We remark that establishing (42) for all $k$, implies $f_{r}(2 k)=O\left(r^{k(k-1)}\right)$ for all $k$ by the same argument. 


\section{REFERENCES}

[1] J. Balogh, R. Morris, and W. Samotij, Independent sets in hypergraphs, J. Amer. Math. Soc. 28 (2015), no. 3, 669-709, DOI 10.1090/S0894-0347-2014-00816-X. MR3327533 $\uparrow 2$

[2] C. T. Benson, Minimal regular graphs of girths eight and twelve, Canad. J. Math. 18 (1966), 1091-1094. MR0197342 (33 \#5507) 66

[3] T. F. Bloom, A quantitative improvement for Roth's theorem on arithmetic progressions, available at arXiv:1405.5800. submitted. $\uparrow 1.2$

[4] J. A. Bondy and P. Erdős, Ramsey numbers for cycles in graphs, J. Combinatorial Theory Ser. B 14 (1973), 46-54. MR0317991 (47 \#6540) $\uparrow 6$

[5] J. A. Bondy and M. Simonovits, Cycles of even length in graphs, J. Combinatorial Theory Ser. B 16 (1974), 97-105. MR0340095 (49 \#4851) $\uparrow 6$

[6] P. Erdős, On sequences of integers no one of which divides the product of two others and on some related problems, Mitt. Forsch.-Inst. Math. und Mech. Univ. Tomsk 2 (1938), 74-82. $\uparrow 6$

[7] _ Extremal problems in graph theory, Theory of Graphs and its Applications (Proc. Sympos. Smolenice, 1963), Publ. House Czechoslovak Acad. Sci., Prague, 1964, pp. 29-36. MR0180500 (31 \#4735) $\uparrow 6$

[8] P. Erdős, Problems and results on finite and infinite graphs, Recent advances in graph theory (Proc. Second Czechoslovak Sympos., Prague, 1974), Academia, Prague, 1975, pp. 183-192. MR0389669 (52 \#10500) $\uparrow 1.1,1.3$

[9] _ Problems and results in combinatorial number theory, Journees Arithmétiques de Bordeaux (Conf., Univ. Bordeaux, Bordeaux, 1974), Soc. Math. France, Paris, 1975, pp. 295-310. Astérisque, Nos. 24-25. MR0374075 (51 \#10275) $\uparrow 1.2$

[10] P. Erdős and R. L. Graham, On partition theorems for finite graphs, Infinite and finite sets (Colloq., Keszthely, 1973; dedicated to P. Erdős on his 60th birthday), Vol. I, North-Holland, Amsterdam, 1975, pp. 515-527. Colloq. Math. Soc. János Bolyai, Vol. 10. MR0373959 (51 \#10159) $\uparrow 1.1$

[11] P. Erdős and A. Hajnal, Reserach Problems, Acta Math. Acad. Sci. Hungar 17 (1966), 61-99. MR0193025 (33 \#1247) 11.2

[12] _ Research problems 2-5, J. Combinatorial Theory 2 (1967), 104-105. $\uparrow 1.3$

[13] P. Erdős and M. Simonovits, Compactness results in extremal graph theory, Combinatorica 2 (1982), no. 3, 275-288, DOI 10.1007/BF02579234. MR698653 (84g:05083) $\uparrow 6,6$

[14] J. Folkman, Graphs with monochromatic complete subgraphs in every edge coloring., SIAM J. Appl. Math. 18 (1970), 19-24. MR0268080 (42 \#2979) $\uparrow 1.3$

[15] R. L. Graham and J. Nešetřil, Large minimal sets which force long arithmetic progressions, J. Combin. Theory Ser. A 42 (1986), no. 2, 270-276, DOI 10.1016/0097-3165(86)90097-X. MR847557 (88c:11015) $\uparrow 1.2$

[16] S. Janson, T. Łuczak, and A. Rucinski, Random graphs, Wiley-Interscience Series in Discrete Mathematics and Optimization, Wiley-Interscience, New York, 2000. MR1782847 (2001k:05180) ^3, 4, 5

[17] R. Nenadov and A. Steger, A short proof of the random Ramsey theorem, Combin. Probab. Comput. 25 (2016), no. 1, 130-144, DOI 10.1017/S0963548314000832. $\uparrow 2,5$ 
[18] J. Nešetřil and V. Rödl, The Ramsey property for graphs with forbidden complete subgraphs, J. Combinatorial Theory Ser. B 20 (1976), no. 3, 243-249. MR0412004 (54 \#133) $\uparrow 1.3$

[19] _ Van der Waerden theorem for sequences of integers not containing an arithmetic progression of $k$ terms, Comment. Math. Univ. Carolinae 17 (1976), no. 4, 675-681. MR0441906 (56 \#297) 11.2

[20] Simple proof of the existence of restricted Ramsey graphs by means of a partite construction, Combinatorica 1 (1981), no. 2, 199-202, DOI 10.1007/BF02579274. MR625551 (83a:05101) $\uparrow 1.3$

[21]__ Sparse Ramsey graphs, Combinatorica 4 (1984), no. 1, 71-78, DOI10.1007/BF02579159. MR739415 (85h:05073) 11.3

[22] V. Rödl, On Ramsey families of sets, Graphs Combin. 6 (1990), no. 2, 187-195, DOI 10.1007/BF01787730. MR1073689 (91m:05137) $\uparrow 1.2$

[23] V. Rödl and A. Ruciński, Threshold functions for Ramsey properties, J. Amer. Math. Soc. 8 (1995), no. 4, 917-942, DOI 10.2307/2152833. MR1276825 (96h:05141) $\uparrow 1.1,1.2$

[24] V. Rödl, A. Ruciński, and M. Schacht, An exponential-type upper bound for Folkman numbers, Combinatorics. to appear. $\uparrow 2,3,5,5$

[25] T. Sanders, On Roth's theorem on progressions, Ann. of Math. (2) 174 (2011), no. 1, 619-636, DOI 10.4007/annals.2011.174.1.20. MR2811612 (2012f:11019) $\uparrow 1.2$

[26] D. Saxton and A. Thomason, Hypergraph containers, Invent. Math. 201 (2015), no. 3, 925-992, DOI 10.1007/s00222-014-0562-8. MR3385638 $\uparrow 2,2$

[27] R. Singleton, On minimal graphs of maximum even girth, J. Combinatorial Theory 1 (1966), 306-332. MR0201347 (34 \#1231) $\uparrow 6$

[28] J. Spencer, Restricted Ramsey configurations, J. Combinatorial Theory Ser. A 19 (1975), no. 3, 278-286. MR0382058 (52 \#2946) 11.2

Instituto de Matemáticas, Pontificia Universidad Católica de Valparaíso, Valparaíso, CHILE

E-mail address: han.hiep@googlemail.com

Department of Mathematics and Computer Science, Emory University, Atlanta, USA

E-mail address: $\{$ tretter I rodl \}@mathcs.emory.edu

Fachbereich Mathematik, Universität Hamburg, Hamburg, Germany

E-mail address: schacht@math.uni-hamburg.de 